\title{
Use of an electronic system in the surveillance of adverse events following immunization (AEFI) in Sri Lanka: A pilot study
}

\author{
A.K.J.M. Wijayarathne ${ }^{1 *}$, M.M.N.S. Dilan ${ }^{1}$, W.M.R.R. Wickramarathne ${ }^{1}$, S. Ginige ${ }^{2}$ \\ 1 Post Graduate Institute of Medicine, Colombo, Sri Lanka \\ 2 Epidemiology Unit, Ministry of Health and Indigenous Medicine, Sri Lanka \\ *jayathriwijayarathne@gmail.com \\ https://orcid.org/0000-0003-0840-3288 \\ ABSTRACT
}

\begin{abstract}
Introduction: The Expanded Programme on Immunization (EPI) established in 1978 'in Sri Lanka, has continued to make excellent progress over the past four decades, most notably in terms of achieving high immunization coverage and disease control. When the perception of risk associated with vaccine preventable diseases decreases, the fear of adverse effects following immunization (AEFI) tend to increase. This can reduce compliance with vaccination, allowing for the re-emergence of controlled diseases. AEFI reporting is important as it is a direct measure of the efficiency of the immunization programme in a country. In Sri Lanka, a solid system of paper-based reporting is in place. AEFI systems are aimed at providing information that allow for continuous assessment of the safety of a given vaccine in the studied population in a timely manner. The objective of this study was to analyse the requirements of AEFI system and to develop a web-based system to report data from divisional and regional areas to the Epidemiology Unit.
\end{abstract}

Methods: The study was conducted in two phases. The first phase was to identify the data flow and the existing paper-based formats. In-depth interviews with relevant stakeholders were carried out as the second phase to gather requirements. The data gathered were qualitatively analysed to derive the requirements. Market research was done to identify the most suitable platform/system to cater the requirements. A system was customized using DHIS2 platform. Data sent in paper-based formats to the Epidemiology Unit for the first quarter of year 2019 were entered into the system by data entry operators as a pilot study. The system was then presented and evaluated by the AEFI experts at the Epidemiology Unit to see whether it derives the 'data for action'.

Results: A set of requirements were derived as a result of qualitative analysis. The stakeholders prioritized the need to collect aggregate AEFI data island wide. Hence, DHIS2 platform was identified as suitable for the purpose and customized according to the needs of the users. the stakeholders accepted that the system derives the needed data and the data quality was better in the electronic system compared to the existing paper-based format.

Conclusions: The DHIS2 based electronic system was suitable to capture AEFI. The developed system will be used countrywide at the Medical officer of Health level and regional levels to collect and report AEFI data to the provincial and national level. A future study should be done to evaluate the scalability of the system to record individual incidents at the clinic or field level.

Keywords: Adverse Events Following Immunization, Electronic AEFI surveillance, e-AEFI system, Electronic Immunization Information System 